\title{
QUALIDADE DE VIDA SOB A ÓTICA DE ENFERMEIROS DO CENTRO CIRÚRGICO DE UM HOSPITAL PÚBLICO
}

Daniela Aires Cardoso dos Santos 1 Dalyanne Souza Vieira Diniz Morais ${ }^{2}$ Renata Valero Barbosa Franco ${ }^{1}$ Jacqueline Ramos de Andrade Antunes Gomes ${ }^{3}$ http://orcid.org/0000-0002-4443-9117 http://orcid.org/0000-0002-8457-4196 http://orcid.org/0000-0001-7734-2832 http://orcid.org/0000-0002-7243-4073

Objetivos: Identificar e descrever as percepções de enfermeiros atuantes no centro cirúrgico de um hospital público do Distrito Federal quanto à qualidade de vida. Metodologia: Estudo descritivo com abordagem qualitativa. A coleta de dados foi por meio de entrevista semiestruturada, com amostra de 11 enfermeiros, no período de setembro de 2016 a janeiro de 2017. Os depoimentos foram transcritos e analisados pela técnica de análise de conteúdo, por meio da qual as evocações foram organizadas e agrupadas em categorias. Resultados: Após a análise, emergiram os temas: percepção da qualidade de vida e qualidade de vida no trabalho. Conclusões: Observou-se a qualidade de vida como um termo polissêmico, tendo relação direta com o trabalho. A pesquisa visa fornecer subsidios para a formulação de estratégias em benefício da qualidade de vida dos profissionais.

Descritores: Qualidade de vida; Saúde do trabalhador; Centro cirúrgico.

\section{QUALITY OF LIFE FROM THE POINT OF VIEW OF NURSES FROM THE SURGICAL CENTER OF A PUBLIC HOSPITAL.}

Objectives: To identify and describe the perceptions of nurses working in the surgical center of a public hospital in the Federal District regarding quality of life. Methodology: Descriptive study with a qualitative approach. Data collection was done through a semistructured interview with a sample of 11 nurses, from September 2016 to January 2017. The testimonies were transcribed and analyzed by the content analysis technique, through which the evocations were organized and grouped into categories. Results: After the analysis, the themes emerged: perception of quality of life and quality of life at work. Conclusions: Quality of life was observed as a polysemic term, having a direct relation with the work. The research aims to provide subsidies for the formulation of strategies to benefit the quality of life of professionals.

Descriptors: Quality of life; Worker's health; Surgical center.

\section{CALIDAD DE VIDA BAJO LA ÓPTICA DE ENFERMEROS DE CENTRO QUIRÚRGICO DE UN HOSPITAL PÚBLICO.}

Objetivos: Identificar y describir las percepciones de enfermeros actuantes en el centro quirúrgico de un hospital público del Distrito Federal en cuanto a la calidad de vida. Metodología: Estudio descriptivo con abordaje cualitativo. La recolección de datos fue por medio de entrevista semiestructurada, con muestra de 11 enfermeros, en el período de septiembre de 2016 a enero de 2017. Los testimonios fueron transcritos y analizados por la técnica de análisis de contenido, por medio de la cual las evocaciones fueron organizadas y agrupadas en categorías. Resultados: Después del análisis, surgieron los temas: percepción de la calidad de vida y calidad de vida en el trabajo. Conclusiones: Se observó la calidad de vida como un término polisémico, teniendo relación directa con el trabajo. La investigación pretende proporcionar subsidios para la formulación de estrategias en beneficio de la calidad de vida de los profesionales.

Descriptores: Calidad de vida; Salud del trabajador; Centro quirúrgico. 


\section{INTRODUÇÂO}

De acordo com a Organização Mundial da Saúde (OMS), a qualidade de vida pode ser compreendida como o nível de percepção que o indivíduo tem diante da sua posição na vida, inclusive sua cultura, expectativas, preocupações e valores materiais $^{(1)}$.

O termo é utilizado pela sociedade para descrever necessidades subjetivas e objetivas na busca de promover o equilibrio interno e externo(1). Em vários contextos, é possível observar que a qualidade de vida é usada como sinônimo de saúde. Porém, afirmar que ambas são sinônimas é o mesmo que simplificá-la a um único fragmento de bem-estar da integralidade humana ${ }^{(2)}$.

O trabalho é um fator que está associado diretamente na vida do indivíduo, pois é o meio pelo qual se procura satisfazer suas aspirações ${ }^{(3)}$. A vivência da qualidade de vida no trabalho está presente quando são supridas as seguintes necessidades pessoais: apoio institucional; segurança, integração e satisfação com o seu papel no trabalho; percepção do bemestar obtido através do seu trabalho e do desenvolvimento pessoal alcançado; e administração de seu tempo livre ${ }^{(4)}$.

Em relação à enfermagem, tem-se que ela exige de seus profissionais disponibilidade, sensibilidade e conhecimento científico para cuidar do outro de forma integral. Ainda que para exercer a profissão seja necessária boa saúde física e mental, os enfermeiros nem sempre desempenham suas funções sob condições necessárias de proteção e atenção ${ }^{(5)}$.

Os trabalhadores de saúde, destacando a enfermagem, se deparam comosentimento defrustraçãoe descontentamento devido a elevada responsabilidade do exercício profissional e a prática recorrente de duplicidade laboral, gerando sobrecarga de trabalho que ocasiona transtornos físicos, psicológicos e sociais, interferindo na relação de trabalho e na sua qualidade de vida ${ }^{(6,7)}$.

O local de trabalho pode estar relacionado como um dos fatores estressores para os profissionais de enfermagem. $\bigcirc$ Centro Cirúrgico (CC) é um setor que possui características peculiares por ser um setor fechado, a execução do trabalho com postura inadequada e a necessidade de a equipe de enfermagem dar suporte aos demais membros da equipe cirúrgica, além da inadequação do número de trabalhadores, baixa remuneração e a capacitação inadequada dos profissionais para o uso de novas tecnologias, fazem deste setor um local que interfere na qualidade de vida destes profissionais $^{(6)}$.

Sabendo que os fatores que interferem na qualidade de vida podem comprometer o cuidado prestado, faz-se necessário conhecê-los para que mudanças necessárias ocorram afim de promover o bem-estar dos mesmos, assim como a adequação de condições para a sua reabilitação( ${ }^{(8)}$.
Conhecer os fatores que levam ao prazer e ao sofrimento dos profissionais de enfermagem é o passo inicial para conduzir o trabalho de forma produtiva, colaborativa e mais humana, evitando doenças físicas e psíquicas relacionadas ao sofrimento no trabalho(9).

Para tanto, é essencial realizar novos estudos, pois segundo a literatura, não há estudos suficientes direcionados a esse grupo de trabalhadores relacionando ao centro cirúrgico, uma vez que o estresse é uma condição presente na rotina profissional(6).

Considerando esta tela sobre a qual se projetam as vivências do profissional que atua em centro cirúrgico, pretendeu-se com este estudo identificar e descrever as percepções de enfermeiros atuantes no centro cirúrgico de um hospital público do Distrito Federal quanto à qualidade de vida. Neste contexto, a pesquisa busca responder à seguinte pergunta: Quais são as percepções dos enfermeiros atuantes no centro cirúrgico de um hospital público do Distrito Federal a respeito do conceito de qualidade de vida?

\section{METODOLOGIA}

\section{Tipos de estudo}

Trata-se de uma pesquisa descritiva com abordagem qualitativa.

\section{Participantes da pesquisa}

A amostra foi de 11 enfermeiros, de uma população de 20 profissionais. Como critério de inclusão, estabeleceu-se que o individuo deveria ser enfermeiro assistencial atuante no centro cirúrgico do hospital sede do estudo e que concordasse em participar da pesquisa, de ambos os gêneros, de qualquer faixa etária. Como critério de exclusão, definiuse: profissionais não lotados na unidade - tanto residentes, estagiários e internos de cursos de graduação, quanto profissionais de outros setores que fazem horas extras no setor, e também enfermeiros coordenadores da unidade, assim como, sujeitos que estivessem de licença ou em férias.

\section{Local do estudo}

Realizada no centro cirúrgico do hospital de base do Distrito Federal (DF). É um hospital público de alta complexidade que atende toda a população do DF, entorno e estados circunvizinhos. O centro cirúrgico é composto por dezesseis salas cirúrgicas, sendo duas separadas para cirurgias de emergência.

\section{Coleta de dados}

A coleta de dados foi por meio de entrevista semiestruturada e a seleção dos entrevistados foi feita de forma aleatória e voluntária. 
As entrevistas foram realizadas em sala reservada na instituição, nos períodos matutino, vespertino e noturno gravadas em aparelho de mp4, até a saturação do conteúdo das falas dos participantes com duração média de 35 a 40 minutos. A entrevista tratou de temas específicos sobre qualidade de vida, resumindo-se a três perguntas: "O que é qualidade de vida?", "Como você avalia a sua qualidade de vida?" e "O que poderia melhorar na sua qualidade de vida?". O período de coleta de dados foi entre 29 de novembro e 10 de dezembro de 2016.

\section{Procedimentos de análises de dados}

Os depoimentos foram transcritos e analisados pela técnica de análise de conteúdo, sendo esta uma técnica por meio da qual as evocações foram organizadas e agrupadas em categorias para analisar as afirmações com maior clareza ${ }^{(10)}$. Desta análise emergiram os temas: percepção sobre qualidade de vida e qualidade de vida no trabalho. Cada unidade de análise está identificada com um elemento alfanumérico que codifica os sujeitos por ordem das entrevistas. O participante 1, por exemplo, foi identificado como El, onde "E" representa a palavra "Enfermeiro" e o "l" identifica o sujeito. Ao final de cada entrevista, os participantes foram questionados se gostariam de desistir ou se queriam alterar alguma resposta, mas não foi registrado nenhum caso.

\section{Procedimentos éticos}

O estudo foi realizado seguindo as recomendações éticas emitidas pela Resolução do Conselho Nacional de Saúde no 466 de 12/12/2012(11), que aborda a pesquisa em seres humanos, respaldado nos aspectos éticos e legais. A pesquisa foi aprovada pelo Comitê de Ética em Pesquisa da Fundação de Ensino e Pesquisa em Ciências da Saúde (FEPECS), sob número do CAAE 60429816.6.0000.5553, conforme parecer 1.850.936, na data de 17 de novembro de 2016.

\section{RESULTADOS}

Após análise técnica do conteúdo coletado, as evocações foram organizadas e agrupadas em categorias para analisar as afirmações. Desta análise emergiram os temas: percepção sobre qualidade de vida e qualidade de vida no trabalho.

\section{Percepção sobre qualidade de vida}

Estiveram presentes nas falas termos como saúde, atividade física, hábitos saudáveis, prazer, lazer como se vê a seguir: É um conjunto de coisas. Tem a ver com saúde, tem a ver com família e tem a ver com prazer no emprego. Tendo essas três coisas balanceadas, se complementando, acho que é uma boa qualidade de vida (E2). Para mim, é viver em família (...), e além disso é ter condição de fazer uma atividade física, boa alimentação, ter condição de ter uma saúde estabelecida (E4). Comer bem, fazer exercício físico, o que eu não estou fazendo (E3). Hoje eu coloquei na minha vida o exercício físico. Isso melhorou minha qualidade de vida consideravelmente. Atividade física, dedicar mais tempo para a família, estar mais com Deus, e aí isso tudo tem me feito estar bem. (ElO)

Ao serem questionados sobre como avaliam a própria qualidade de vida, os participantes deram respostas que variaram entre péssima, mediana e boa. Eu acho que a minha qualidade de vida é mediana (El). A minha qualidade de vida é boa, eu consigo fazer tudo (E9). A minha qualidade, ela é péssima (...) (E7).

\section{Qualidade de vida no trabalho}

Os entrevistados relacionam prazer na profissão assim como em outros âmbitos da vida: Gosto de trabalhar, mas não gosto de viver em função do trabalho (E4). A gente precisa gostar daquilo que faz, vir para o trabalho com prazer. Eu sou enfermeira por opção, amo minha profissão (E8).

A multiplicidade de fatores que interferem na qualidade de vida dos indivíduos abre margem para que este conceito se relacione também com as condições de trabalho: Qualidade de vida é ter condições de trabalho (E7). Significa que você tenha boas condições no ambiente de trabalho. Condições humanas, condições de material, condições de recursos humanos, recursos materiais (E8).

A respeito das más condições de trabalho, os participantes relatam: Na falta de pessoal, eu vou ter que entrar mais ainda ajudando na equipe de enfermagem. Então o desgaste físico e psicológico dobra. Isso repercute na minha vida profissional: o meu cansaço e o estresse psicológico (E7).

Os ganhos salariais são tidos pelos profissionais entrevistados como um importante componente da qualidade de vida: Eu preciso ganhar na mega sena para poder melhorar minha vida, ter mais grana para poder viver (El). Eu acho que eu ganho razoavelmente bem, eu tenho de certa forma conforto em casa, então isso contribui para minha qualidade de vida (Ell)

O duplo vínculo empregatício em busca de maiores recursos financeiros esteve presente nas falas dos participantes, como é possivel observar na seguinte fala: Se você ficar empolgado só num salário aqui em Brasília, para você assumir a família, não dá, nunca deu, pelo menos para mim nunca deu. Por isso que eu trabalhei em dois (E9).

A sobrecarga laboral repercute na qualidade de vida, segundo as falas dos participantes: O trabalho da enfermagem é muito estressante. O período que eu tenho de descanso, que é para a família, para o lazer, acaba ficando para o descanso do trabalho, que é muito cansativo (E2). 


\section{DISCUSSÃO}

Em relação ao conceito polissêmico de qualidade de vida, embora admitindo como um termo difícil de definir, os participantes reconheceram o seu caráter multifatorial e admitiram que diversas dimensões compõem este conceito.

Não há consenso sobre o termo qualidade de vida, mas sabe-se que há muitos fatores individuais na definição do termo, como: o ambiente físico e suas condições, a estrutura familiar e financeira, a saúde, educação e cultura ${ }^{(12)}$.

Embora o termo qualidade de vida resulte em uma visão individual e de suas necessidades, a saúde, o trabalho e a familia são dimensões de grande relevância à vida dos trabalhadores. Vale salientar que possuir qualidade de vida engloba diversos aspectos que vão muito além do trabalho, no entanto deve haver incentivo afim de proporcionar um ambiente humanizado, o que acarreta em diminuição no absenteísmo e nos acidentes de trabalho, e proporciona um ambiente saudável ${ }^{(3)}$.

Por meio das falas dos participantes sobre a percepção sobre qualidade de vida foi possivel inferir que variadas dimensões da vida sofrem influência das condições de trabalho, repercutindo negativamente a avaliação da mesma pelo indivíduo.

A falta de tempo para o lazer e à recreação também se relacionou à fadiga, estando diretamente ligada aos erros no desempenho do trabalho e ao desenvolvimento de um ambiente inseguro para oferta de cuidados ${ }^{(4)}$. Os profissionais reconhecem a importância de dedicar tempo aos hábitos saudáveis, mas a ausência de tempo compromete não somente a vida social do indivíduo e a sua saúde, mas também reduz a segurança do cuidado ao paciente.

Dentre os entrevistados, é perceptível que embora haja prazer, há também a vontade de que, além do trabalho, outros âmbitos componham a própria vida. A literatura evidenciou que os profissionais de enfermagem estão parcialmente satisfeitos com sua qualidade de vida no trabalho, e esta possui desdobramentos que excedem o ambiente de trabalho. Afirma ainda que quando os profissionais de enfermagem exercem as suas atividades laborais em organizações com boas condições estruturais e com profissionais qualificados para o exercício da função, ocasiona uma elevação em sua qualidade de vida(4).

Desta forma, percebe-se que formular programas e ações favorecendo a melhoria da qualidade de vida no ambiente de trabalho da enfermagem proporciona o aumento da eficácia organizaciona( ${ }^{(4)}$. Como resultado, a melhoria no trabalho leva a uma assistência de saúde mais adequada e qualificada ao paciente alvo do cuidado ${ }^{(13)}$.

É sabido que a qualidade de vida, sendo indissociável do trabalho, sofre interferência direta deste, visto que ele pode potencializar a saúde ou o adoecimento, com danos sobre a integridade física e/ou psicológica, afirmando ainda que a falta de planejamento das atividades e a condição de trabalho em ambiente confinado intensificam a vivência do estresse no setor do centro cirúrgico ${ }^{(6,14)}$. Os participantes alegaram que a falta de recursos humanos e materiais causa cansaço e estresse, e que a disponibilidade de adequadas condições de trabalho poderiam favorecer o bem-estar.

Os ganhos salariais são um fator preponderante no que diz respeito às condições de trabalho transformando em vantagens para a vida do trabalhador, sendo um benefício advindo do trabalho representando uma motivação. É por meio do dinheiro que as pessoas organizam e planejam suas vidas, atendem suas necessidades materiais e atingem os seus objetivos financeiros ${ }^{(3)}$. Sabe-se que a enfermagem ainda é uma profissão mal remunerada no Brasil. Os baixos salários da categoria foram apontados como uma das causas de maior insatisfação profissional. Por este motivo, a maioria dos trabalhadores da enfermagem se vê forçada a trabalhar em mais de um local, ocasionando a permanência destes profissionais no ambiente de trabalho durante a maior parte do tempo de suas vidas produtivas ${ }^{(13)}$. O duplo vínculo empregatício e a sobrecarga laboral repercutem na qualidade de vida, pois nos periodos em que não estão trabalhando, os profissionais não têm disposição para fazer outra coisa além de descansar.

No decorrer do exercício das atribuições do profissional, a sobrecarga de trabalho favorece a ocorrência de acidentes no ambiente de trabalho, bem como de agravos a saúde que poderiam ser identificados e prevenidos antecipadamente ${ }^{(7)}$.

Sentir-se eficiente também influencia positivamente o bem-estar do individuo, sendo esse um fator preponderante do processo saúde-doença em sua relação com o trabalho (12). Sentir-se útil no trabalho é uma recompensa moral(3). É como se o tempo dispendido na preparação para o trabalho e na própria realização deste fosse pago com satisfação pessoal e sensação de utilidade.

\section{Limitações do estudo}

Com a realização desta pesquisa, percebe-se o quão limitado são os estudos que envolvem o tema qualidade de vida no trabalho na área da enfermagem, dificultando a busca no acervo de referências bibliográficas. Os resultados devem ser interpretados observando as experiências de cada profissional e considerar que a pesquisa foi realizada com poucos profissionais

\section{Contribuições do estudo}

Desta forma, almeja-se promover enriquecimento em tal assunto a fim de despertar novos estudos e confere ao profissional de enfermagem um aprofundamento no tema. 


\section{CONCLUSÃO}

Osentrevistadosadmitiramotermo qualidadedevidacomo polissêmico, sendo a relação com o trabalho preponderante nesta pesquisa. O prazer decorrente da profissão foi citado como fator necessário ao bom desenvolvimento do trabalho, consequentemente contribuindo para uma boa qualidade de vida. Sentir-se útil no trabalho é a recompensa moral trazida pelo mesmo, influenciando positivamente o desempenho profissional e a organização do trabalho.

Participar de um estudo como este possivelmente favoreceu aos indivíduos a autorreflexão a respeito do estilo de vida que tem sido adotado, e dos seus desdobramentos na própria vivência diária, nos seus mais diversos aspectos.

\section{Contribuição dos autores}

Daniela Aires Cardoso dos Santos:Concepção e/ou desenho, análise e interpretação dos dados, redação do artigo, revisão crítica e revisão final;Dalyanne Souza Vieira Diniz Morais:Revisão crítica e revisão final; Renata Valero Barbosa Franco; Revisão crítica e revisão final;Jacqueline Ramos de Andrade Antunes Gomes ;Concepção e/ou desenho, análise e interpretação dos dados, redação do artigo, revisão crítica e revisão final.

\section{REFERÊNCIAS}

1. Freire MN, Costa ER. Qualidade de vida dos profissionais de enfermagem no ambiente de trabalho. Rev. enfermagem contemporânea [internet]. $2016 \mathrm{mar} /$ jul [cited $2018 \mathrm{mar} 19]$ ] 5(1). Available from: ht tps://www5.bahiana.edu.br/index.php/enfermagem/article/view/871 tps://www5.bahiana.edu.br/index.php/enfermagem

2. Moura IH de, Nobre R de S, Cortez RMA, Campelo V, Macêdo SFde. Silva ARV da. Quality of life of undergraduate nursing students. Rev. Gaúcha Enferm [Internet]. 2016 mai [cited 2018 mar 14]; 37(2). Available from: http://www.scielo.br/scielo.php?script=sci_arttext\&pid=S1983-14472016000200407\&lng=en\&nrm=iso\&tlng=en . http://dx. doi.org/10.1590/1983-1447.2016.02.55291.

3. Dias EG, Santos AR dos, Souza ELS, Araújo MML, Alves JCS, Mishima SM. Qualidade de vida no trabalho dos profissionais da saúde de uma Unidade Básica de Saúde. Rev. Cubana de enfermeria [internet]. 2016 jan [cited 2018 mar 15]; 32(4). Avaliable from: http://www.revenfermeria.sld.cu/index.php/enf/article/view/1075

4. Zavala MOO, Klinj TP, Carrillo KLS. Quality of life in the workplace for nursing staff at public healthcare institutions. Revista Latino-Americana de Enfermagem [Internet]. 2016 ago [cited 2016 nov 30]: 24 Available from: http://dx.doi.org/10.1590/1518-8345.1149.2713

5. Carvalho A de MB, Cardoso JA, Silva FAA da, Lira JAC, Carvalho SM. Qualidade de vida no trabalho da equipe de enfermagem do centro cirúrgico. Rev. Enferm. Foco [Internet]. 2018 [Cited 2018 dez 12]: 9(3): 3541. Available from: http://revista.cofen.gov.br/index.php/enfermagem/ article/view/1159/458

6. Jacques JPB, Ribeiro RP, Martins JT, Rizzi DS, Schmidt DRC. Geradores de estresse para os trabalhadores de enfermagem de centro cirúrgico. Semina: Ciências Biológicas e da Saúde [Internet]. 2015 ago [cited $2018 \mathrm{abr}$ 15]; 36(1): 25-32. Available from: https://www.researchgate net/publication/283887589 Geradores_de_estresse_para os trabalhadores de enfermagem de_centro_cirurgico. http:// os_trabalhadores_de_enfermagem

7. Karino ME, Feli VEA, Sarguis LMM, Santana LL, Silva SR, Teixeira RC Cargas de trabalho e desgastes dos trabalhadores de enfermagem de um hospital-escola. Cienc. Cuid. Saúde [Internet]. 2015 Abr/Jun [cited 2018 abr 16]; 14(2):1011-1018. Available from: http://periodicos.uem.
br/ojs/index.php/CiencCuidSaude/article/view/21603 . http://dx.doi. org/10.4025/cienccuidsaude.v14i2.21603.

8. Marques ALN, Ferreira MBG, Duarte JMG, Costa N dos S, Haas VJ, Simões AL de A. Quality of life and working context of nursing professionals of the Family Health Strategy. Revista Rene [Internet]. 2015 mai/ago [ cited 2016 nov 29]: 16(5). Available from: https://doi. org/10.15253/2175-6783.2015000500008.

9. Inoue KC, Versa GLG da S, Júnior JAB, Murassaki ACY, Matsuda LMM. Qualidade de vida e no trabalho de enfermagem: revisão integrativa da literatura. Revista uningá review [internet]. 2018 jan [cited 2018 mar 18]: 16(1). Available from: http://revista.uninga.br/index.php/uningareviews/article/view/1452

10. Silva AH, Fossá MIT. Análise de conteúdo: exemplo de aplicação da técnica para análise de dados qualitativos. Qualitas Rev. Eletrônica [Internet]. 2015 [cited 2018 abr 15];17 (1). Available from: http://dx.doi. org/10.18391/qualitas.v16il.2113

11. Resolução 466, de 12 de dezembro de 2012 (BR). Aprova as diretrizes e normas regulamentadoras de pesquisas envolvendo seres humanos. Diário Oficial da União[Internet], Brasilia (DF). 13 jun 2013 [cited 1 dez 2016]. Available from: http://conselho.saude.gov.br/resolucoes/2012/ Reso466.pdf

12. Lopes CCP, Ribeiro TP, Martinho NJ. Sindrome de Burnout e sua relaça com a ausência de qualidade de vida no trabalho do enfermeiro. Rev. Enfermagem em foco [internet]. 2012 [cited 2018 mar 16]: 3(2): ro. Rev. Enfermagem em foco [internet]. 2012 [cited 2018 mar 16]; 3(2):
97-101. Available from: http://revista.cofen.gov.br/index.php/enfer97-101. Available from: http:/

13. Moreira WC, Ferreira AA, Sousa ARR de, Santos WN dos, Silva HLL da, Costa GR da, et al. Night Work And Its Implications In The Quality Of Life Of Nurses. International Archives of Medicine [Internet]. 2017 mar [cited 2018 mar 14]; 10. Available from: http://imedicalsociety.org/ ojs/index.php/iam/article/view/2280 . https://doi.org/10.3823/2364 .

14. Sampaio CL, Barros LM, Neri MFS, Neto NMGN, Frota NM, Do Nascimento JC, et al. Nurses' Self-Evaluation of Health and Quality of Life Inside the Workplace. International Archives of Medicine [Internet]. 2017 abr [cited 2018 mar 14]: 10. Available from: http://imedicalsociety.org/ ojs/index.php/iam/article/view/2323. https://doi.org/10.3823/2367. 